\title{
Interface entre le verbal et le non verbal: Comprendre le mécanisme de la violence verbale en classe
}

\author{
Christina Romain $^{1}$, Brahim Azaoui ${ }^{2}$ et Véronique Rey ${ }^{3}$

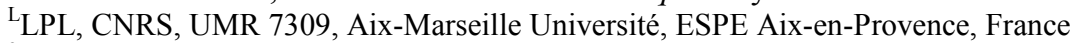 \\ ${ }^{2}$ LIRDEF EA 3749, Université de Montpellier / Université Paul Valéry, France \\ ${ }^{3}$ CREDO CNRS UMR 7308 Aix-Marseille Université, ESPE Aix-en-Provence, France
}

\begin{abstract}
Résumé. A partir d'une analyse linguistique interactionnelle de deux échanges entre enseignant et élève, nous avons questionné l'articulation entre les dimensions verbale et non verbales d'une montée en tension verbale jusqu'à sa réalisation physique. L'analyse verbale a été conduite à travers l'étude des actes de langage et de leur organisation, tandis que l'analyse non verbale a été menée en considérant différentes réalisations mimogestuelles (regard, mimiques, gestes coverbaux) et proxémiques des interactants.
\end{abstract}

\begin{abstract}
Interface between verbal and non verbal dimensions : Understanding the mechanism of verbal violence in class. Based on an interactive linguistic analysis of two exchanges between teachers and pupils, we questioned the articulation between the verbal and non verbal dimensions of a verbal rise in tension to a physical realization. The verbal analysis was conducted through the study of speech acts and their organization, while the non-verbal analysis was carried out by considering different mimogestural (gaze, mimics, cospeech gestures) and proxemic accomplishments of the interactions.
\end{abstract}

Le sujet de la violence verbale apparait comme un sujet d'une actualité criante dans notre société actuelle, comme le rappellent les débats autour du rapport entre les sexes, des agressions verbales et physiques, voire dans le cadre du terrorisme (cf. projet H2020 Partnership Against The Radicalization in the Cities (PRACTICIES) dont le Workpackage 4 porte sur les discours de haine et leur performativité). Les travaux qui lui sont consacrés tout particulièrement depuis une dizaine d'années ont permis de modéliser la montée en tension (Fracchiolla et al., 2013) et de l'appliquer au contexte éducative (voir notamment Romain et Rey, $2016 \mathrm{ab}$ ). A ce jour toutefois, aucune étude n'a cherché à analyser l'interface entre la dimension verbale et posturo-mimogestuelle dans cette montée en tension. Or, cette compréhension est nécessaire pour qui veut saisir les enjeux de ce

\footnotetext{
${ }^{1}$ Corresponding author : christina.romain@univ-amu.fr
} 
processus dans l'interaction prise dans sa globalité, en incluant donc ce qui relève du non verbal.

A partir d'une analyse de deux extraits vidéo d'échanges entre enseignant et élève en classe, nous avons questionné l'articulation entre les dimensions verbale et posturomimogestuelle d'une montée en tension verbale jusqu'à sa réalisation physique. L'analyse verbale a été conduite à travers l'étude des actes de langage et de leur organisation, tandis que l'analyse non verbale a été menée en considérant différentes réalisations mimogestuelles (regard, mimiques, gestes coverbaux) et proxémiques des interactants.

Les résultats montrent, selon les situations vidéo analysées, comment la montée en tension est fondamentalement une réalité multimodale. Dans la première vidéo, l'aspect verbal finit par refléter la tension impliquée par un processus de territorialisation permis par le comportement posturo-mimogestuel de l'enseignante. Dans le second extrait, la désescalade du conflit est permise par la gestion verbale de la montée en tension et par la place du regard. Celui-ci n'évoque pas la recherche de l'affrontement, mais la reconnaissance de l'autre comme son interlocuteur.

\section{Cadre théorique}

\subsection{La description de la montée en tension verbale : type de montée en tension, acte de langage et taxèmes de positionnement}

Nous avons commencé par analyser l'interaction verbale au regard de l'analyse du type de montée en tension verbale à partir du modèle élaboré par Fracchiolla et al. (2013) et aménagé pour les interactions pédago-didactiques par les travaux de Romain et Rey (2016ab). Ces auteures décrivent différents type de violence verbale pouvant s'alimenter les uns et les autres : la violence verbale fulgurante marquée par un ou des déclencheurs de conflits accompagnés d'actes de langage dépréciatifs directs ; la violence verbale polémique marquée par une argumentation à visée polémique (à charge d'autrui) ; la violence verbale argumentative marquée par une argumentation à visée coopérative (coopération et co-énonciation); la violence verbale détournée à visée de polémique consistant en une sur-énonciation d'un point de vue dominant à travers le recours à des actes de langage indirects ; la violence verbale détournée à visée argumentative convoquant des actes de langage indirects visant la persuasion par la raison.

Ces différents types de montée en tension convoquent différents actes de langage (désormais AL) (Austin : 1962; Searle : 1969; Romain et Lorenzi : 2013) : les AL modestes caractérisés par la place laissée aux atténuateurs de menace ; les AL ardents de second degré reposant sur une menace argumentée visant une relation positive entre enseignant et élève (fonctionnement systématisé de l'interaction pédago-didactique de la classe) ; les AL ardents de premier degré présentant un caractère exclusivement menaçant (ex. : ton carnet!). Les AL modestes et les AL ardents de second degré se croisent avec la montée en tension (détournée) argumentative tandis que les AL ardents de premier degré se croisent avec la montée en tension fulgurante et la montée en tension (détournée) polémique à charge.

Dans cette perspective d'analyse, il a été observé que deux formes de taxèmes de positionnement pouvaient être identifiées (Romain et Lorenzi : 2013) : les taxèmes à valeur de position (marqueurs de relation dissymétrique, autoritariste voire agonale) ; et les taxèmes à valeur de relation (marqueurs de relation coopérative et consensuelle). La notion de face (Goffman: 1974) et celle de politesse linguistique en tant que vectrice de 
conciliation voire de négociation interactionnelle (Lakoff : 1973 ; Brown et Levinson : 1987 ; Leech : 1983 ; Kerbrat-Orecchioni : 1992) permettent de mieux comprendre l'enjeu de l'interaction verbale et de conduire à l'identification des outils linguistiques favorisant une interaction pédagogique consensuelle et coopérative. Les outils de politesse linguistique visent tant à atténuer les AL menaçants qu'à favoriser les AL rassurants pour la relation interdiscursive et la relation interpersonnelle (taxèmes de relation). Inversement, les outils d'impolitesse linguistique visent à produire des actes menaçants pour la face sans atténuateur significatif (taxèmes de position).

Notre propos s'inscrit dans le cadre de ces études en se focalisant sur la compréhension de la montée en tension verbale en classe, en croisant les rapports entre type de montée en tension, AL et politesse linguistique ; mais aussi, et ce sera l'objet de notre second point théorique, de prendre en compte la mimogestualité constitutive de la montée en tension verbale.

\subsection{L'analyse mimogestuelle de la montée en tension verbale}

Les réflexions menées sur la question du rapport à l'espace dans les travaux sur la proxémie menés par Hall (1971) ou sur la mimogestualité (Argyle et al. : 1972 ; Watzlawick et al. : 1972 ; Kendon : 1967 ; McNeill : 1992) permettent d'accroitre la compréhension que nous avons des interactions humaines.

Dans une étude anthropologique de l'espace, Hall a mis au jour l'existence de quatre différentes distances interindividuelles (intime, personnelle, sociale et publique). Il a ainsi montré l'effet de l'organisation et de la gestion de ces espaces sur les interactions quotidiennes et interculturelles. Les études sur la proxémie en classe sont encore peu nombreuses, malgré quelques travaux précurseurs menés il y a une trentaine d'années (De Landsheere et Delchambre: 1979; Pujade-Renaud: 1983). Plus récemment, Moulins rappelle que «c'est d'abord par ses déplacements dans la classe, que le maître affirme sa présence » (2004: 146). Dans le prolongement des conclusions de Hall, l'auteur souligne que « se rapprocher ainsi d'un élève c'est symboliquement entrer dans son territoire, c'est envahir sa sphère privée ; ce qui n'est pas sans conséquence sur l'état émotionnel de l'élève et sur son comportement »[ibid]. Nous nous apercevrons dans l'analyse de notre corpus que ces éléments sont cruciaux pour comprendre les enjeux de la montée en tension.

Pour ce qui est de la mimogestualité, nous la considérons comme un élément constitutif du discours. Les modalités de communication forment un tout et sont interdépendantes. Nous rejoignons en cela Krafft et Dausendschön-Gay (2001 : 120), qui défendent une « conception holistique des phénomènes langagiers et colangagiers ».

L'enjeu toutefois ne se limite pas à repérer les réalisations non verbales de la communication, mais bien d'en saisir leur implication au niveau des échanges. Kendon (2004 : 158-159) envisage une fonction modale de la gestuelle dans la mesure où elle vient modifier l'interprétation à donner à l'énoncé. Le geste modalise le discours en exprimant, selon Calbris et Porcher (1989 : 190) : « une réaction positive (de joie) ; négative, active (de colère ou d'énervement par exemple) ou passive (impuissance, désolation). Vis-à-vis de l'interlocuteur, le geste est susceptible d'exprimer l'attendrissement (pour s'excuser, quémander), la volonté de rassurer ou au contraire, de façon négative : le dénigrement, la menace, l'avertissement... De l'attitude, on passe au commentaire, positif ou négatif, ne serait-ce qu'en mettant en valeur ou en minimisant l'énoncé ».

On retrouve ces mêmes valeurs dans les études sur les mimiques faciales, et notamment celles sur le regard. Ces travaux (Kendon : 2004 ; Goodwin : 1981 ; Heylen : 2005) ont montré les nombreuses fonctions que le regard remplit sur le plan cognitif ou interpersonnel.

Dans une perspective plus éthologique, le détournement du regard est souvent perçu comme une tentative, chez les animaux, de diminuer l'agression ou comme un rituel 
d'apaisement. Cosnier (2015) évoque une étude dans laquelle les participants décrivent les mécanismes de protection pour contrôler ce qu'ils disent. Parmi ceux-ci, l'on retrouve l'évitement ou la fuite que permet le détournement du regard. Il permet aussi d'affirmer sa position tout en restreignant le risque de sentiment de menace et de dominance (Corraze : 1980).

Dans le contexte pédagogique, ces phénomènes ont fait l'objet de travaux par Foerster (1990), ou encore Azaoui, qui les a traités dans le cadre d'une réflexion sur les normes interactionnelles (2015a), mettant au jour des implications sur le plan énonciatif (2015b). Son analyse de la montée en tension en classe de français langue première et en français langue seconde (Azaoui : 2014) a permis de souligner le rôle du non verbal dans la gestion des rapports de place (Flahault : 1978).

\section{Méthodologie}

Dans le cadre de ce travail, nous allons analyser deux échanges tirés de deux corpus vidéo distincts : le premier est un extrait vidéo intitulé «Opposition au travail demandé » disponible sur l'environnement de formation NéoPass@ction (IFE) (cf. le programme de recherche et de formation à l'origine du développement de ressources pour la formation : Leblanc et Ria : 2014). On y observe la fin d'un cours d'histoire-géographie avec des élèves de troisième. Le second provient du corps qu'Azaoui a collecté dans le cadre de son travail doctoral (Azaoui : 2014). L'enseignante dispense un cours de français langue seconde à des élèves allophones dont la maitrise des normes scolaires en France est en construction. Ces deux échanges donnent à voir une montée en tension verbale entre une enseignante et un élève au collège.

Nous avons procédé d'une part à l'analyse interactionnelle des échanges en prenant en compte le type de montée en tension verbale, les AL et les formes de politesse et d'impolitesse linguistiques qui la caractérisent. D'autre part, nous nous sommes intéressés à des indicateurs d'analyse de la mimogestualité. Nous avons considéré différentes réalisations multimodales, mais avons prêté une attention particulière aux jeux de proxémie et de regard, qui nous ont semblé assez symptomatique des enjeux de l'échange à ce niveau d'analyse.

\section{Analyse des résultats}

\subsection{De la cristallisation de la montée en tension verbale à la violence physique}

\subsubsection{Analyse verbale}

L'exemple que nous nous proposons d'analyser est constitutif d'une montée en tension fulgurante, détournée à visée de polémique et enfin polémique à charge. IL a retenu notre attention car il dure plusieurs minutes et donne à voir différentes stratégies discursives de l'enseignante et de l'élève conduisant la montée en tension verbale jusqu'à son paroxysme : la violence physique (l'élève frappant le mur).

Nous retranscrivons ci-après l'échange analysé :

Ce sont les dernières secondes de l'heure de cours. L'enseignante est au tableau puis elle s'approche de l'élève E1 et regarde son travail.

1. P : Si t (u) as rien souligné, tu vas pas sortir ++ Eh t(u) as vu comment tu m'as noté ? Et tu crois que je vais te laisser sortir comme ça?

2. E1 : Ouais

3. P : Oh oui, bien sûr... 


\section{E1 : (rire)}

(L'enseignante retourne au tableau. Elle regarde de loin le cahier de l'élève et reviens près de lui regarder à nouveau son travail, les bras croisés sur la poitrine.)

5. $\mathrm{P}: \mathrm{J}(\mathrm{e})$ sais pas mais on va demander (L'enseignante produit un geste de la main sur le cahier.) au reste de la classe (L'enseignante prend le cahier et le montre à la classe.) A votre avis quand on a copié comme ça, est ce qu'on peut sortir?

6. E2 : Ouah, c'est gros

7. P : Avec moi, on sort ? Avec ça ? + Non

8. E1 : Mais j'arrive à lire moi !

(L'enseignante repose le cahier sur la table de l'élève, qui le reprend. Ils se disputent le cahier. Elle le reprend finalement.)

9. P : Donc, hop, donne-moi ça, + donne-moi ça

(L'élève lit alors une partie de son texte.)

10. E1 : $\mathrm{xxx} \mathrm{J}(\mathrm{e})$ sais lire

(L'enseignante tourne les pages du cahier et déchire une page.)

11. E1 : allez moi j'me barre

12. P : Tu recommences!

(L'élève se lève en même temps.)

13. E1 : Non j(e) m barre.

14. P : Pardon?

15. E1 : non j(e)m casse.

(L'enseignante se place devant la porte, les bras croisés, tandis que l'élève se lève et range sesaffaires. L'enseignante, bras croisés, s'appuie alors contre la porte un peu négligemment/relâchée.)

16. $\mathrm{E} 1: \mathrm{J}(\mathrm{e}) \mathrm{m}$ barre c'est tout $++\mathrm{xxx}$ Je réécris rien du tout.

17. $\mathrm{P}:$ Ben eh t'avais qu'à écrire correctement du premier + du premier coup.

18. E1 : Je réécris rien du tout.

19. $\mathrm{P}: \mathrm{J}$ 'en n'ai rien à faire ! + Tu vas voir si tu réécris rien du tout...

20. E1 : Non

21. P : Si si

22. E1 : Non

23. P Si

24. E1 : Non

(L'élève range ses affaires pendant cet échange et va poser son cahier au fond de la classe).

25. $\mathrm{P}$ (toujours devant la porte) : Donne ton cahier Kévin $++\mathrm{Tu}$ te dépêches (L'élève revient devant la porte et face à l'enseignante sans le cahier. Il tente de sortir.) ++ donne ton cahier! Tu sortiras pas!

26. E1 : ben si je sors

27. P. : tu sortiras pas

28. E1 : non non

29. P. : t'as pas fait ton travail/ t'as pas fait ton travail correctement

30. E1 : ben si je l'ai fait

31. P. : non/ va t'asseoir correctement/ tous les autres ils ont dit/ non

32. E1 : non/ ben $\mathrm{si}<\ldots$ ? >

33. P. : ben eux c'est bien fait/ tu sortiras pas

(L'élève continue de ranger ses affaires et remonte sa fermeture éclair, met son sac à dos.)

34. E1. : si je sors

35. P. : non

36. E1. : si

37. P. : non

38. P. : tu t'assoies 
(L'élève met sa capuche.)

39. P. : non non

40. E1. : je sors

41. P. : tu t'assoies

42. E1. : non

(L'élève range sa chaise.)

43. P. : tu t'assoies

(L'enseignante remet la chaise à sa place pour que l'élève reprenne sa place, alors que celui-ci se dirige vers la porte.)

44. P. : tu ne sors pas (L'enseignante positionne la chaise sur le côté de la table, l'élève ne peut plus reprendre "sa » place. Une fois la chaise posée, l'enseignante reprend sa position, le regard fixe orienté vers E1, bras croisés. Elle ne le quitte pas du regard lui alors que l'élève a le regard fuyant.)

Silence : 9 secondes

45. P. : (L'enseignante produit un geste déictique caractérisé par un bras tendu et un regard orienté vers le fond de la classe.) va t'asseoir

46. E1 : bien sûr (L'élève regarde l'enseignante en souriant-sourire initié plus tôt.)

47. P. : VA T'ASSEOIR (L'enseignante le tire légèrement et brièvement par la veste.) tu te dépêche (Elle produit un geste déictique plus petit, bref et sec par rapport au précédent.) va t'asseoir

48. E1. : non

(L'élève reste debout.)

49. P : (L'enseignante retrouve une position bras croisés, regard tourné vers l'élève.) Donc tu vas pénaliser tout le monde alors ++ toi tu sors pas les autres ils sortent pas c'est ça (L'enseignante recherche ses clés tout en maintenant son regard sur l'élève puis elle produit un hochement de tête. Elle produit ensuite un mouvement pour aller vers le bureau mais rebrousse chemin à reculons pour se positionner finalement les bras croisé/dos contre porte.)

50. $\mathrm{P}$ : prends les clés sur la table (En direction d'un autre élève.)

51. P : t'ouvres la porte (L'enseignante produit un geste déictique vers une autre porte située dans le prolongement du tableau -côté opposé à la porte centrale.)

52. E2 : laquelle + celle-là ?

53. P : (Hochement de tête et l'enseignante se positionne au niveau de l'élève -pour prévenir une possible fuite ?) romain tu ouvres la porte et vous sortez

(E1 tente de " prendre la fuite » sans précipitation, " tranquillement ».)

54. $\mathrm{P}:$ tu viens ici

(L'enseignante tente de le rattraper par la veste. Un jeu d'esquive s'engage pour accéder à la porte en premier.)

55. P : non non non non non non hop

(L'enseignante le repousse et l'élève bascule et il se retient au mur.)

56. P : Tu ne sors pas EH!

(L'enseignante le repousse à l'intérieur de la salle de classe.)

57. E1 : ben si j'sors

(L'élève se glisse par un espace entre chambranle et l'enseignante... la situation va s'enliser jusqu'à ce que l'enseignante et l'élève en vienne à une altercation physique: l'élève voulant sortir et l'enseignante cherchant toujours à l'en empêcher et demandant aux autres élèves d'aller chercher quelqu'un pour l'aider.)

58. E1. : lâchez-moi

59. P. : non tu ne sors pas

60. E1. : lâchez-moi putain

61. P. : assieds-toi

62. E1. : mais non j'arrête lâchez-moi putain 
(L'élève prend sa tête dans ses mains, il devient violent contre lui-même, il donne un coup de poing dans le mur...)

A ce stade, nous proposons un tableau de synthèse des tours de parole entre l'enseignante nommée $\mathrm{P}$. et l'élève nommé $\mathrm{E} 1$. Ce tableau vise à répertorier de façon synthétique le face à face entre les tours de parole en termes de formes de montée en tension, afin de mettre en évidence à la fois leur chronologie mais aussi leur répartition entre l'enseignante et l'élève.

Tableau 1. Tours de parole et montée en tension verbale (exemple 1).

\begin{tabular}{|c|c|c|c|}
\hline $\begin{array}{c}\text { Montée en tension } \\
\text { (chronologie des } \\
\text { étapes) }\end{array}$ & $\begin{array}{c}\text { Tours de } \\
\text { parole de } \\
\text { l'enseignante } \\
\end{array}$ & $\begin{array}{c}\text { Tours de } \\
\text { parole de } \\
\text { l'élève } \\
\end{array}$ & Remarques \\
\hline M.T. Polémique & 1 & & $\begin{array}{l}\text { La montée en tension est initiée } \\
\text { par de la polémique à charge (à } \\
\text { l'issue de l'heure de cours). }\end{array}$ \\
\hline $\begin{array}{c}\text { M.T. Détournée à } \\
\text { visée de polémique }\end{array}$ & & 2 & \\
\hline $\begin{array}{l}\text { M.T. Détournée à } \\
\text { visée de polémique }\end{array}$ & 3 & & \\
\hline $\begin{array}{l}\text { M.T. Détournée à } \\
\text { visée de polémique }\end{array}$ & & 4 & \\
\hline M.T. Polémique & $5 ; 7$ & & $\begin{array}{l}\text { Ici l'enseignante prend à témoin le } \\
\text { groupe classe. }\end{array}$ \\
\hline M.T. Argumentative & & 8 & $\begin{array}{l}\text { L'élève est le seul interactant à } \\
\text { convoquer de la tension } \\
\text { argumentative (argumentation par } \\
\text { la raison). Il réagit en se défendant } \\
\text { (alors que lors de ses premières } \\
\text { interventions il ne dit quasiment } \\
\text { rien). }\end{array}$ \\
\hline M.T. Fulgurante & 9 & & $\begin{array}{l}\text { L'enseignante convoque la tension } \\
\text { fulgurante : refus de recourir à } \\
\text { l'argumentation, injonction de } \\
\text { faire. }\end{array}$ \\
\hline M.T. Argumentative & & 10 & $\begin{array}{l}\text { A l'issue du tour de parole } 10, \\
\text { l'enseignante déchire la feuille du } \\
\text { cahier de l'élève. A partir de là, la } \\
\text { relation interdiscursive bascule } \\
\text { dans une relation } \\
\text { frontale/fulgurante de la part de } \\
\text { l'élève. }\end{array}$ \\
\hline M.T. Fulgurante & 12 & $11 ; 13$ & \\
\hline $\begin{array}{l}\text { M.T. Détournée à } \\
\text { visée de polémique }\end{array}$ & 14 & & \\
\hline M.T. Fulgurante & & $15 ; 16$ & L'élève refuse toutes les \\
\hline
\end{tabular}




\begin{tabular}{|c|c|c|c|}
\hline & & & $\begin{array}{l}\text { injonctions en bloc tandis que } \\
\text { l'enseignante recourt sans succès à } \\
\text { la polémique }\end{array}$ \\
\hline M.T. Polémique & 17 & & \\
\hline M.T. Fulgurante & $\begin{array}{c}19 ; 21 ; 23 ; \\
25 ; 27\end{array}$ & $\begin{array}{l}18 ; 20 ; \\
22 ; 24 ; \\
26 ; 28\end{array}$ & $\begin{array}{l}\text { Elève et enseignante recourent à } \\
\text { une montée en tension fulgurante } \\
\text { (miroir) }\end{array}$ \\
\hline M.T. Polémique & 29 & & \\
\hline M.T. Fulgurante & & 30 & \\
\hline M.T. Polémique & 31 & & \\
\hline M.T. Fulgurante & & 32 & \\
\hline M.T. Polémique & 33 & & $\begin{array}{l}\text { L'enseignante recourt à la } \\
\text { polémique mais l'élève reste dans } \\
\text { la fulgurance }\end{array}$ \\
\hline M.T. Fulgurante & $\begin{array}{l}35 ; 37 ; 38 ; \\
39 ; 41 ; 43 ; \\
\quad 44 ; 45\end{array}$ & $\begin{array}{l}34 ; 36 ; \\
40 ; 42\end{array}$ & $\begin{array}{l}\text { L'enseignante le rejoint dans la } \\
\text { fulgurance (miroir) }\end{array}$ \\
\hline $\begin{array}{l}\text { M.T. Détournée à } \\
\text { visée de polémique }\end{array}$ & & 46 & \\
\hline M.T. Fulgurante & 47 & 48 & \\
\hline M.T. Polémique & 49 & & \\
\hline M.T. Fulgurante & $\begin{array}{c}54 ; 55 ; 56 ; \\
59 ; 61\end{array}$ & $\begin{array}{c}57 ; 58 ; \\
60 ; 62\end{array}$ & $\begin{array}{l}\text { Procédé identique : l'enseignante } \\
\text { tente de recourir à de la polémique } \\
\text { (argumentation à charge) mais } \\
\text { l'èlève reste dans la fulgurance où } \\
\text { elle va très rapidement le rejoindre } \\
\text { à nouveau. La voie } \\
\text { interactionnelle est sans issue et } \\
\text { conduit à la violence physique } \\
\text { (l'élève se fait physiquement } \\
\text { violence, l'enseignante ouvre alors } \\
\text { la porte pour le protéger de lui- } \\
\text { même selon son propre } \\
\text { témoignage) }\end{array}$ \\
\hline
\end{tabular}

Cet échange est constitutif d'une montée en tension fulgurante initié par de la tension polémique à charge survenant à l'issue d'un exercice intervenant en fin de cours. Alors que l'enseignante relève, en prenant la classe à témoin, l'imperfection de la réalisation d'un exercice produit par un des élèves, celui-ci conteste le fait de devoir recommencer l'exercice que l'enseignante cherche à lui imposer. Il range ses affaires et tente de sortir de la classe tandis que les autres élèves en font autant, la fin de l'heure de cours étant atteinte. Il s'agit là probablement d'un des principaux moteurs de la tension verbale intervenant lors de cet échange.

On observe que cet échange se structure autour de plusieurs points de cristallisation de la tension :

- L'enseignante signifie tout d'abord à l'élève son mécontentement quant au travail réalisé. L'enseignante initie un registre discursif polémique à charge tandis que l'élève convoque un registre discursif détourné à visée de polémique à charge (il tente d'esquiver le conflit). 
- L'élève ne réagissant pas comme elle l'attend, l'enseignante va prendre à témoin la classe. L'élève entre alors dans une production argumentative pour assurer sa défense. Mais l'enseignante refuse d'entrer à son tour dans de l'argumentation, elle poursuit l'échange dans un registre polémique à charge.

- L'enseignante déchire alors le travail de l'élève et lui interdit de quitter la salle sans que le travail ne soit refait. Elle initie alors un registre discursif fulgurant auquel l'élève répond de manière égale et continue jusqu'à l'issue de l'échange. On notera que l'enseignante tente de varier son registre d'attaque, mais elle le fait sans jamais chercher une coopération voire un début de négociation argumentée. Il est intéressant d'observer ici que certains éléments restent dans le non-dit. L'enseignante se contentant de signifier à l'élève que l'on ne quitte pas son cours en ayant fait un «tel» travail et lui enjoint de recommencer. A aucun moment, elle ne prend en compte le cadre de la classe et les horaires qui régissent l'emploi du temps. A aucun moment non plus, elle ne prend en compte explicitement l'attente du travail: on comprend que l'élève n'a pas écrit "correctement» mais que signifie le «correctement »? Il s'agit là d'un subjectivème péjoratif mais qui n'est pas accompagné d'une explicitation objective.

A partir du moment où l'enseignante déchire le travail réalisé, l'élève entre donc en tension verbale fulgurante et y reste jusqu'à l'issue de l'échange. L'enseignante va tenter à deux reprises de redescendre dans un registre polémique à charge mais elle finit par recourir à chaque fois à de la tension fulgurante. Les deux registres fulgurants (de l'élève et de l'enseignante) se répondent en miroir, tandis que l'élève répond au registre polémique de l'enseignante par un registre invariablement fulgurant. Il est intéressant ici d'observer que le comportement mimogestuel et proxémique de l'élève ne s'aligne pas sur la tension fulgurante observable sur le plan verbal. Cette divergence est remarquable dans sa réalisation de ses gestes : alors que le verbal est tout en fulgurance, les gestes manuels et les déplacements sont lents, précis, consciencieux (il range calmement ses affaires et soigne le matériel de la classe sans précipitation). Il ne reconnait pas l'injonction de l'enseignante (refaire son travail) mais continue à respecter son rôle d'élève par rapport au temps scolaire (ranger ses affaires et sortir de la classe à l'issue de l'heure de cours).

Concernant la politesse linguistique, on observe qu'elle est largement absente du registre discursif de l'enseignante. Celle-ci attaque la face de l'élève systématiquement alors que l'élève de son côté tente d'atténuer au maximum la tension naissante et intense qui prend corps dans l'échange : il répond de façon implicite, il tente de convoquer des éléments objectifs (la lisibilité voire la visibilité de son écrit), il repousse la tension fulgurante jusqu'à la matérialisation de cette tension par l'enseignante (elle déchire le cahier et tente de le contraindre physiquement de rester dans la salle). Ainsi, l'enseignante ne cherche à aucun moment à négocier et la tension s'attise d'elle-même : l'élève conteste dans un premier temps l'injonction (ironie) puis, lorsque l'enseignante déchire son travail, il la refuse systématiquement. Toutefois, il continue à respecter son rôle d'élève conformément à ce que font les autres élèves : il range son cahier sur l'étagère, il range ses affaires dans son sac, il range sa chaise sur son bureau, il met et ferme son blouson et il se dirige vers la sortie de la classe. Il est intéressant d'observer ici qu'il continue à se comporter conformément aux autres élèves (c'est la fin de l'heure de cours, les élèves rangent leurs affaires et se dirigent vers la sortie). Il repousse le conflit verbal par la tension fulgurante et demande à ce qu'il cesse, à sortir de la salle.

On observe la réalisation d'une impasse interdiscursive : l'enseignante monte en tension, avive et ravive la tension sans efficacité sur le comportement attendu de l'élève (il ne refait pas son travail, il ne veut pas rester dans la classe). Pourtant l'enseignante insiste et met en danger la relation interdiscursive. Elle poursuit sa logique d'attaque jusqu'à recourir à la violence matérielle et à la contrainte physique (elle déchire la feuille du cahier, elle cherche à contraindre l'élève à rester, à fermer la porte de la classe et à en barrer physiquement l'accès). L'enseignante convoque de façon répétitive une majorité d'AL 
menaçants ardents de premier degré. Elle initie la montée en tension verbale par de la fulgurance («tu vas pas sortir») et de la polémique à charge («t(u) as rien souligné, $t(u)$ as vu comment tu m'as noté ? et tu crois que je vais te laisser sortir comme ça?»). Cette montée en tension s'enrichit et se renforce par d'autres AL menaçants ardents de premier degré indirect ( oh oui, bien sûr») et des AL menaçants ardents de second degré directs à visée polémique à charge («j(e) sais pas mais on va demander au reste de la classe/ à votre avis quand on a copié comme ça/ est-ce qu'on peut sortir? et encore avec moi, on sort? avec ça? + non »).

L'élève de son côté va tenter des justifications via l'argumentation visant la persuasion («mais j'arrive à lire moi!») qui restent sans effet («tu recommences!, j'en ai rien à faire! + tu vas voir si tu réécris rien du tout, si, tu t'assoies, va t'asseoir VA T'ASSEOIR tu te dépêche va t'asseoir »). L'élève répondant à son tour par des AL menaçants ardents de premier degré ( «non, non j(e)m casse, j(e)m barre c'est tout $++j e$ réécris rien du tout, si je sors ») jusqu'à ce que la tension verbale culmine par le recours à la contrainte physique de l'enseignante ( lâchez-moi putain »).

Finalement, les AL menaçants de l'enseignante sont inefficaces puisque l'élève lui oppose un refus systématique de faire. Malgré des tentatives de variation dans la forme de montée en tension (polémique à charge et détournée), l'enseignante ne cherche pas à négocier et reste positionnée dans un registre discursif agonal. La montée en tension atteint finalement son paroxysme par le recours à la violence physique de l'élève dirigée contre lui-même.

Au final, ce type de montée en tension, poussée à son paroxysme, conduit à la violence physique (ici l'élève retourne cette violence contre lui-même en prenant sa tête entre ses mains et en la tapant contre le mur).

\subsubsection{Analyse posturo-mimogestuelle}

L'analyse posturo-mimogestuelle, qui implique la gestion du corps et de ce qui relève des gestes manuels et des mimiques/regards,montrera comment la montée en tension se couple d'une territorialisation (notion que nous empruntons au champ scientifique de la géographie qui l'entend comme l'action exercée sur un espace pour se l'approprier (Raffestin : 1986)) de la classe de la part de l'enseignante, qui vise en cela à organiser et à maitriser l'espace physique. Différentes étapes de la montée en tension illustrent ce processus.

Ainsi, lorsque l'enseignante se rapproche de l'élève pour observer, voire inspecter son travail, elle pénètre dans son espace physiquement et visuellement. Elle arrive les bras croisés et avance d'un pas décidé vers le bureau de l'élève. Elle accentue son "intrusion » spatiale en jetant un regard sur le cahier de l'élève (Fig.1). L'enseignante a littéralement un droit de regard sur le cahier, qui devient rapidement la source de la montée en tension polémique située au tour de parole 1.

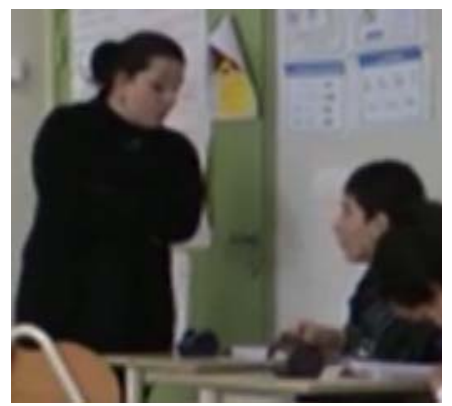

Fig. 1. Regard orienté sur le cahier de l'élève. 
Elle se saisit du cahier de l'élève pour en faire la preuve irréfutable du bienfondé de son argument, mais s'approprie également l'espace de l'élève en s'appuyant sur le bureau. Fait remarquable, au fur et à mesure que l'enseignante soulève le cahier du bureau, l'élève effectue un mouvement de recul pour lui céder la place. Il finit adossé contre le mur, elle appuyée sur le bureau (Fig.2). Le processus de d'appropriation de l'espace scriptural et physique est complet : le cahier et le bureau de l'élève lui appartiennent.
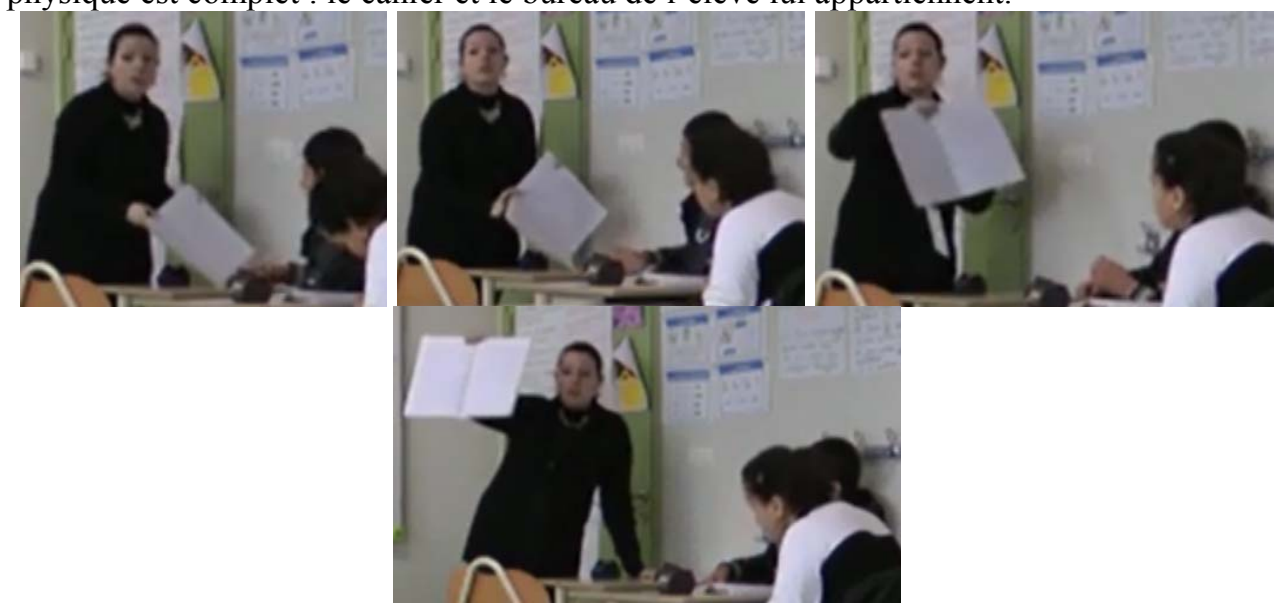

Fig. 2. Synchronie des mouvements posturaux.

Comme signalé plus haut, le tournant dans le rapport de force se situe au moment où l'enseignante s'apprête à déchirer une page du cahier de l'élève. Il se lève alors pour récupérer son sac qui se trouve aux pieds de l'enseignante et «se barrer » (tour de parole 16). Pour cela, il doit contourner son bureau et rejoindre l'enseignante de l'autre côté. Elle n'est plus protégée par le bureau, et doit partager son espace avec l'élève. Dès lors, l'enseignante va chercher à préserver son espace en repoussant l'élève du bras (Fig.3) avant d'adopter une position relâchée, adossée contre la porte (Fig.4).

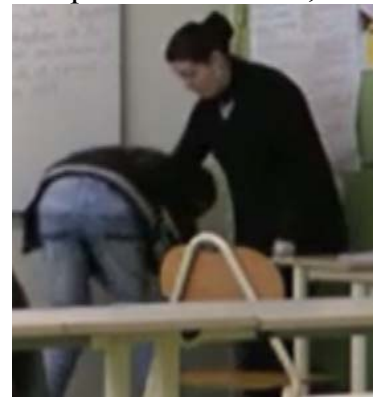

Fig. 3. L'enseignante repousse l'élève

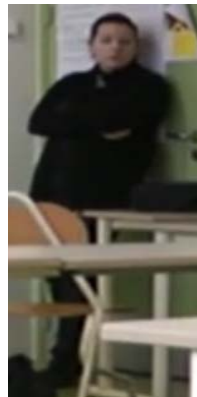

Fig. 4. L’enseignante adossée contre la porte.

Cette posture, que l'on pourrait qualifiée de détendue, est d'autant plus remarquable qu'elle contraste avec la tension verbale et la polémique à charge par l'enseignante. Cette disjonction réalisation verbale/posturale interroge et peut donner lieu à plusieurs interprétations. Tout d'abord, elle fait écho à certains travaux, rapportés par Corraze (1980 : 132), qui rappellent que si l'émetteur a un statut supérieur, «il est alors détendu et sa relaxation est forte ». C'est un peu cette image que semble vouloir donner l'enseignante ; elle est certaine de ce qu'elle avance et de son bon droit. Elle n'a donc pas à s'inquiéter. Une autre interprétation se fonde sur la position des jambes, légèrement avancées, qui ferment l'accès à l'espace intime (Hall : 1971) de l'enseignante. Or, puisque l'enseignante est adossée à la porte, ce même espace intime correspond à celui qui mène à la porte. Aussi, si l'élève souhaitait véritablement forcer le chemin, il serait contraint 
d'abord de marcher sur les pieds de son enseignante. Ainsi, c'est une double enceinte qui, symboliquement, interdit l'accès à la sortie : la porte, qui est fermée, et, le corps de l'enseignante, lui-même fermé par les bras croisés. Dès lors, l'enseignante se positionne comme maitre d'un territoire qu'elle délimite et contrôle.

A l'inverse, il existe parfois une convergence notable entre les productions verbales et non verbales. Dans la logique du cadre théorique présenté précédemment, il est intéressant de voir comment le registre discursif plénipotentiaire, autoritariste de l'enseignante se retrouve non verbalement à plusieurs niveaux: regard, posture et proxémie. Nous pouvons en effet observer un jeu de regards fixes du côté de l'enseignante, qui exercent une forme de dominance sur l'élève, que l'on retrouve dans la fulgurance et les polémiques à charge notamment. Cette analyse est confortée par les travaux d'Argyle et Ingham (1972) qui ont montré que plus la distance se réduit, moins il devrait y avoir d'échange de regard. Or, la vidéo permet de constater que l'enseignante est dans une autre démarche. La capture d'écran suivante (Fig.5) illustre un exemple de situation durant laquelle l'enseignante maintient longuement ( 5 secondes) son regard sur son élève alors que lui-même est, semble-t-il, dans une forme de fuite.

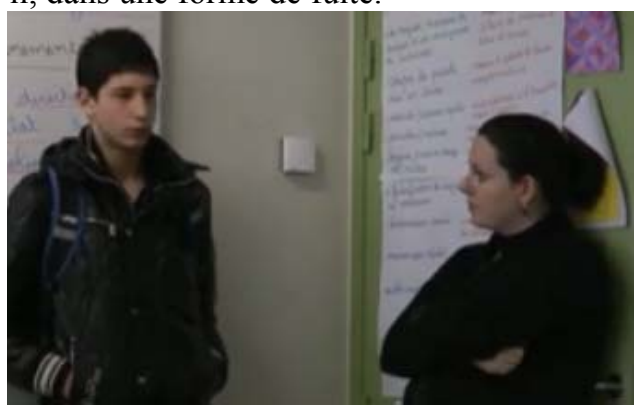

Fig. 5. Regard prolongé de l'enseignante en direction de l'élève.

La pression exercée par l'enseignante se retrouve également dans l'organisation ad hoc de son territoire qu'est la classe. L'élève a rangé sa chaise comme il se doit. Il ne peut donc pas reprendre sa place contrairement à ce que lui enjoint de faire son enseignante. Elle lui en assigne alors une nouvelle, au fond de la classe, qu'elle désigne d'un premier geste déictique ample (Fig.6) dont la réalisation, assez lente, tranche avec la montée en tension fulgurante («va t'asseoir », tour 45).

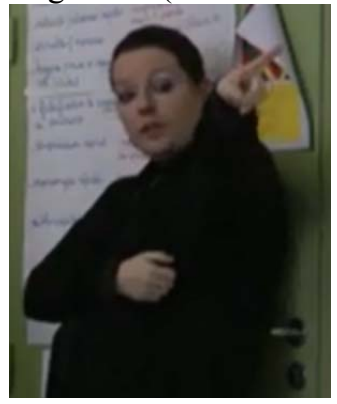

Fig. 6. Assignation verbo-gestuelle de place.

Cette nouvelle assignation suscite un sourire de la part de l'élève qui regarde brièvement l'enseignante (Fig7) : l'enseignante hausse le ton et lui ordonne de s'asseoir (tour de parole 47), le regard toujours orienté vers l'élève. Il est intéressant ici de noter que l'absence de geste déictique est comme compensé par un ton plus ferme, qui pourrait s'expliquer par ailleurs par le sourire moqueur de l'élève. 


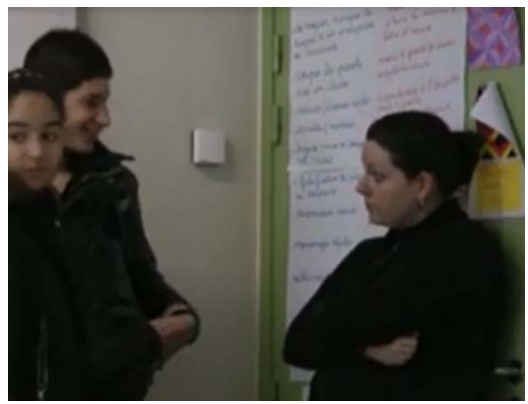

Fig. 7. Sourire de l'élève.

Elle décide alors d'exercer une pression supplémentaire en se saisissant du bout des doigts de la manche de la veste de l'élève pour le déplacer (Fig.8). Elle effectue ensuite un second geste déictique (Fig.9), plus sec, pour lui indiquer l'endroit où il est censé s'asseoir. Notons ici que lors de la réalisation de ces gestes, l'enseignante a le regard tourné vers le sol. Il convient aussi de souligner que c'est le seul moment où il y a un contact physique "prolongé ». La violence symbolique contenue dans ces actes serait-elle compensée/rééquilibrée par un évitement au niveau visuel ?

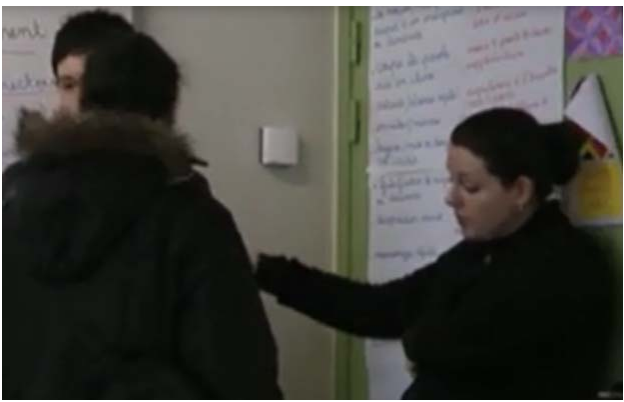

Fig. 8. L'enseignante saisit l'élève par sa veste.

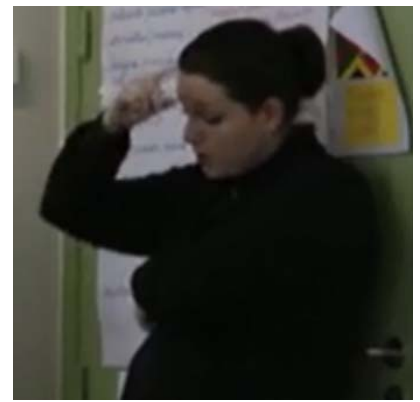

Fig. 9. Geste déictique sec pour assigner la nouvelle place à l'élève.

La suite de l'échange viendrait conforter cette lecture car l'élève tente de sortir par l'autre porte que l'enseignante vient de faire ouvrir par un autre élève (tour de parole 51). Elle le rattrape et doit le retenir en se saisissant à nouveau de sa veste. Or, à ce même moment, son regard est orienté vers le sol et pendant toute la durée du contact physique, elle évitera un contact visuel avec son élève (Fig.10).

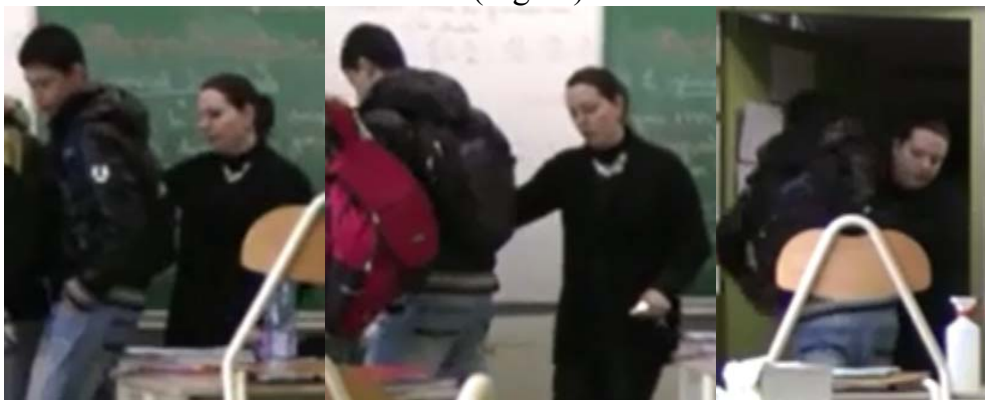

Fig. 10. Contacts physiques et regard au sol.

Fait notable, l'élève demeure les mains dans les poches pendant tout ce contact physique. L'enseignante de nouveau joue de son corps pour interdire l'accès à la sortie en repoussant l'élève au point de le faire tituber. Ce dernier tente alors une première fois de forcer le 
passage, avant de réussir à passer par l'ouverture laissée entre le corps de l'enseignante et le chambranle de la porte.

Ainsi, durant tout cet incident, l'enseignante a cherché à territorialiser sa classe, en l'organisant de telle sorte qu'elle se positionnait comme maitre de cet espace. Ses postures, le regard fixe parfois orienté vers l'élève, les gestes exercés soulignent la place qu'elle entend occuper. Pourtant, si elle s'affirme ainsi même discursivement, l'absence de prise en charge énonciative est remarquable. Nous n'avons comptabilisé que deux occurrences du « je », alors que l'on retrouve essentiellement l'utilisation du pronom « tu », employé dans les polémiques à charge, mais également le pronom « on », dans lequel se perd (ou derrière lequel se cache) l'enseignante.

A l'inverse, si l'élève assume sur le plan énonciatif sa décision, sur le plan non verbal, il rejette l'affrontement que tente de lui imposer l'enseignante. Il est fuyant par le corps et le regard, or, le détournement du regard est souvent perçu comme une tentative de diminuer l'agression ou comme un rituel d'apaisement (Corraze : 1980). Il permet aussi d'affirmer sa position tout en restreignant le risque de sentiment de menace et de dominance. Dans le même temps, cela lui permet de ne pas s'engager dans la négociation puisque l'enseignante disparait littéralement à ses yeux. Sur le plan corporel, il est également dans l'évitement d'abord pour échapper à la main de l'enseignante, qui tente de le rattraper, puis pour ne pas la toucher quand il cherche à passer par la porte.

$\mathrm{Au}$ final, si en surface on a une dominance physique et verbale de l'enseignante, on s'aperçoit qu'elle existe parce que l'élève est essentiellement dans la retenue/évitement. Ironiquement, il nous semble qu'il pousse la retenue jusqu'à finir par retourner la violence contre lui-même.

\subsection{De la cristallisation de la montée en tension verbale à la résolution de la tension}

\subsubsection{Analyse verbale}

A l'inverse du précédent exemple, celui que nous nous proposons d'analyser ici est constitutif d'une montée en tension fulgurante avec une inclusion de tension argumentée à visée de coopération (réparation négociée). Cet exemple a retenu notre attention car il dure une minute et donne à voir des stratégies discursives de l'enseignante et de l'élève qui sont différentes de celles observées dans le premier exemple et qui conduisent à une désescalade de la tension.

Ce qui nous a tout particulièrement intéressé dans cette montée en tension c'est que l'enseignante a un point de vue surplombant à celui de l'élève, elle l'enjoint de changer de place et ne changera pas de positionnement, mais elle laissera un temps d'expression à l'élève, en lui proposant de s'expliquer.

Nous retranscrivons ci-après l'échange analysé :

7. P. : Chut chut les garçons/ non non non non + euh :: $+\mathrm{AXXX}+\mathrm{AXXX} /$ tu viens

t'asseoir ici <mouvement du bras, tendu et index pointé, de l'élève vers la nouvelle place assignée $>$ / dépêche-toi ++ AXXX

8. E1: Ah non madame

9. P. : Pardon <penche le buste>

10. E1 : Non

11. P. : Pourquoi non

12. E1 : Je veux rester là/ parce que c'est ma place

13. P. : Non/ je ne veux pas que tu restes là/ moi $\downarrow<$ regard maintenu, l'élève se lève et change de place, elle le suit du regard, l'élève revient prendre ses affaires -il se dépêche-, 
elle continue à le regarder s'installer à sa nouvelle place $>$ et tu t'assieds correctement/ s'il te plait $\downarrow<$ l'élève termine son installation $>$ voilà $\downarrow+$ alors/ pour le petit journal/ personne 14. E1 : c'est le:: c'est le reportage/ c'est < poursuite du cours auquel l'élève intervient, il reprend son rôle>

A ce stade, comme pour le précédent exemple et avant de rentrer dans le détail de l'analyse, nous proposons un tableau de synthèse des tours de parole entre l'enseignante nommée $\mathrm{P}$. et l'élève nommé E1. Ce tableau vise à répertorier de façon synthétique le face à face entre les tours de parole en termes de formes de montée en tension, afin de mettre en évidence à la fois leur chronologie mais aussi la répartition des formes entre l'enseignante et l'élève.

Tableau 2. Tours de parole et montée en tension verbale (exemple 2).

\begin{tabular}{|c|c|c|c|}
\hline $\begin{array}{l}\text { Montée en tension } \\
\text { (chronologie des } \\
\text { étapes) }\end{array}$ & $\begin{array}{c}\text { Tours de } \\
\text { parole de } \\
\text { l'enseignante }\end{array}$ & $\begin{array}{l}\text { Tours de } \\
\text { parole de } \\
\text { l'élève }\end{array}$ & Remarques \\
\hline M.T. Fulgurante & 7 & & $\begin{array}{l}\text { La montée en tension est initiée } \\
\text { par la fulgurance ( } 2 \text { élèves } \\
\text { discutent entre eux et } \\
\text { l'enseignante enjoint à un des } \\
\text { deux élèves de changer de place). }\end{array}$ \\
\hline M.T. Fulgurante & & 8 & $\begin{array}{l}\text { Refus de l'élève de changer de } \\
\text { place. }\end{array}$ \\
\hline $\begin{array}{l}\text { M.T. Détournée } \\
\text { polémique }\end{array}$ & 9 & & $\begin{array}{l}\text { L'enseignante est surprise et } \\
\text { mécontente, elle produit un AL } \\
\text { indirect menaçant et polémique à } \\
\text { charge « Pardon ?! ». }\end{array}$ \\
\hline M.T. Fulgurante & & 10 & $\begin{array}{l}\text { Réitération du refus par l'élève. } \\
\text { L'élève reste dans la fulgurance. }\end{array}$ \\
\hline M.T. Argumentative & 11 & & $\begin{array}{l}\text { Ici l'enseignante } \\
\text { demande/propose à l'élève de } \\
\text { s'expliquer. Elle quitte la } \\
\text { polémique, revient sur la } \\
\text { fulgurance mais en donnant } \\
\text { l'opportunité à l'élève d'expliquer } \\
\text { son point de vue. } \\
\text { L'argumentation est donc ouverte } \\
\text { par l'enseignante. } \\
\text { A noter : Elle reste sur le premier } \\
\text { nœud de tension (le refus de } \\
\text { changement de place), elle } \\
\text { n'amorce pas une tension sur le } \\
\text { second nœud (l'opposition à son } \\
\text { injonction -potentielle } \\
\text { insubordination, insolence). }\end{array}$ \\
\hline M.T. Argumentative & & 12 & L'élève propose un argument. \\
\hline M.T. Fulgurante & 13 & & $\begin{array}{l}\text { L'enseignante convoque à } \\
\text { nouveau la tension fulgurante : } \\
\text { refus de valider l'argument. Point } \\
\text { de vue surplombant. }\end{array}$ \\
\hline
\end{tabular}


Cet échange est constitutif d'une montée en tension fulgurante initiée par l'enseignante suite au comportement d'un élève discutant avec un autre élève alors qu'elle est en train de poser une question. L'enseignante ordonne à cet élève de changer de place, l'élève refuse. S'il n'y a pas, de la part de l'enseignante de justification explicite (tension fulgurante) à son ordre, le contexte offre la possibilité de comprendre cet acte : les deux élèves sont arrivés en retard sans respecter les usages d'entrée en cours, ce qui a valu un rappel de la part de l'enseignante, et cette dernière les a repris ultérieurement sur leur bavardage et la posture physique de l'un d'eux.

Cet échange est structuré en plusieurs grands temps :

- Le premier nœud de tension explicite : l'enseignante donne l'ordre à l'élève de changer de place sans explication (seul le contexte partagé permet de donner du sens à l'ordre). L'élève va alors refuser sans proposer davantage d'explication.

- L'enseignante initie une tension contournée polémique par le «Pardon ?! » mais l'élève poursuit dans la tension fulgurante (réfutation réitérée).

- L'enseignante initie alors une tension argumentative à visée de négociation/coopération (réparation du nœud de tension). Elle donne la possibilité à l'élève de s'exprimer et de se justifier. Ce qu'il fait : « Je veux rester là parce que c'est ma place ».

- L'enseignante réamorce alors la tension fulgurante en réfutant l'argument «non » et en opposant son propre point de vue « je ne veux pas que tu restes là moi ». Elle ne réitère pas explicitement l'injonction de faire mais elle oppose son point de vue à celui de l'élève. L'injonction reste dans l'implicite mais l'élève s'exécute.

Finalement, les AL menaçants de l'enseignante sont efficaces puisque l'élève, malgré l'opposition systématique du début de l'échange, renonce à la poursuite de la montée en tension. Ce qui est important de souligner ici, c'est qu'il y renonce après avoir eu la possibilité d'argumenter sur son refus. L'enseignante, malgré un point de vue surplombant par lequel elle initie et clôture l'échange, lui propose d'argumenter, de s'expliquer sur son refus. On observe qu'elle initie une tension fulgurante, puis bascule dans une intervention sur la tension détournée à visée de polémique, mais rapidement opte pour proposer une tension argumentative à l'élève. Celle-ci permet à ce dernier de s'exprimer. A la suite de son argumentation, elle oppose un argument « à force de loi » dans la classe «non je ne veux pas que tu restes là/ moi ».

Son refus sera réitéré implicitement néanmoins elle aura adapté la tension au besoin d'expression de l'élève. Elle aura donné la possibilité à l'élève de pouvoir négocier mais finalement son propre point de vue restera premier. Toutefois il est à noter que l'élève a pu opposer librement un argument à l'injonction de l'enseignante qui y aura répondu par un contre argument (certes au demeurant polémique mais qui renvoie implicitement aux rôles et fonctions complémentaires de chacun dans la classe). Elle n'aura pas basculé dans un jeu discursif entre fulgurance et polémique comme pour la première enseignante.

Si son registre discursif apparaît de prime à bord comme agonal, on peut observer qu'il est donc assoupli par le tour de parole proposé à l'élève et par la place laissée à l'argumentation des points de vue de chacun.

$\mathrm{Au}$ final, ce type de montée en tension négociée parvient d'aboutir à une issue positive pour la relation interdiscursive entre enseignant et élève. En effet, comme on le voit à la suite de cet échange, le cours se poursuit et un élève se propose pour répondre à la question posée par l'enseignante.

\subsubsection{Analyse non verbale}

Sur le plan non verbal, l'autorité de l'enseignante s'exprime, tout comme dans l'autre extrait, par un ordre accompagné d'un déictique. Dans les deux cas, le geste est franc : bras et index tendu, et le regard orienté vers le lieu d'assignation (Fig.11) : aucune place n'est donc laissée à un quelconque malentendu. Cependant, dans ce second exemple, 
l'enseignante réoriente son regard vers l'élève (Fig.12), qu'elle reconnait de nouveau comme son interlocuteur principal tout en maintenant, longuement ( 5 secondes), son geste de la main en direction du siège assigné. Elle rétracte son bras au moment où elle prononce « pardon ». Si l'assignation gestuelle est rompue, l'échange en revanche peut se poursuivre. Cette intervention de l'enseignante signale notamment sa surprise d'obtenir une telle réponse. Littéralement, pourrait-on dire, suite à la rétractation du geste déictique, les bras lui en tombent. Sur le plan non verbal, d'ailleurs, son tour de parole est accompagné d'un changement de posture : l'enseignante penche son buste, et de fait, sa tête est penchée en direction de l'élève (Fig.13). Cette modification posturale rend plus explicite le sens à donner à l'interjection de l'enseignante, que l'on pourrait paraphraser ainsi : « répète je ne t'ai pas bien entendu ». C'est d'ailleurs ainsi que l'entend l'élève, qui répète sa réponse précédente.

L'argument faisant «force de loi » dans la classe a raison de l'élève, qui en cela signale son respect, certes relatif, du métier d'élève. Néanmoins, l'enseignante maintient la pression, donc son autorité, en accompagnant du regard les déplacements de cet élève.

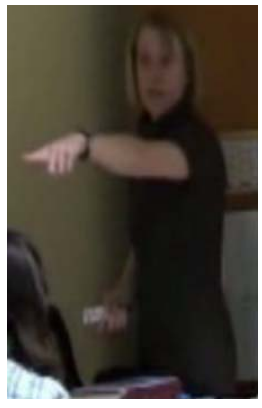

Fig. 11. Geste franc.

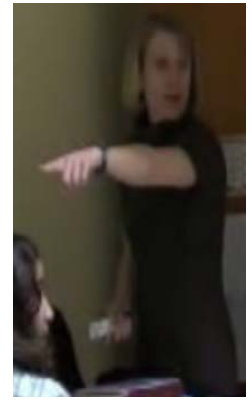

Fig. 12. Réorientation du regard.

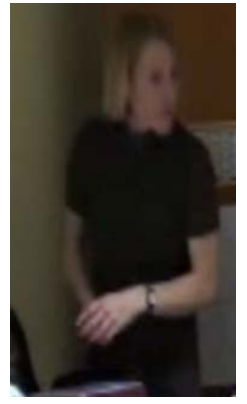

Fig. 13. Changement de posture.

\section{Synthèse}

L'étude verbale et posturo-mimogestuelle a permis de mettre en lumière un certain aspect du processus de montée en tension verbale rendant compte de deux usages différenciés. Dans l'exemple 1, on observe un comportement discursif enseignant focalisé sur un registre discursif agonal (reposant sur de la tension (détournée) polémique à charge et de la tension fulgurante) à l'occasion duquel l'enseignante est aux prises avec les agissements et décisions de son élève qu'elle cherche à cadrer. Dans tous les sens du terme, aucune échappatoire n'est laissée (elle bloque l'entrée, assigne une nouvelle place et retient physiquement l'élève) et il existe une forme de convergence multimodale : l'aspect verbal finit par refléter la tension impliquée par la territorialisation que nous évoquions précédemment. Au contraire, dans l'exemple 2, on observe un processus de montée en tension verbale qui va avoir un effet de désescalade de la tension rendant compte d'un registre discursif davantage coopératif, en recherche de négociation, et au sein duquel l'argumentation occupe une place centrale en ce qu'elle permet d'une part d'expliciter le nœud de tension et d'autre part d'opposer des arguments et non des injonctions et des réfutations. Sur le plan non verbal, , seuls deux gestes, pris au sens large, accompagnent la montée en tension: le geste déictique et le regard. A l'inverse de l'enseignante 1, l'assignation de place n'entraine pas la rupture de la relation interlocutive sur le plan non verbal. Si l'enseignante détache son regard de son élève pour indiquer la place à occuper, ce n'est que sur une brève durée puisqu'elle reprend contact visuellement avec lui. Le regard en direction de l'élève n'évoque pas, ici, la recherche de l'affrontement, mais la 
reconnaissance de l'autre comme son interlocuteur. Les deux enseignantes réagissent donc différemment : si la première refuse toute négociation, tout recours à des stratégies argumentatives, la seconde les initie. Si la tension se cristallise et s'accentue jusqu'à atteindre la violence physique dans le premier exemple, elle s'atténue jusqu'à son extinction définitive dans le second exemple.

En conclusion, notre étude renforce notre connaissance linguistique de la montée en tension verbale en classe à travers ses liens avec la dimension posturo-mimogestuelle. En effet, elle met en avant la différence entre d'une part l'impact d'un usage discursif visant la rupture interactionnelle et l'affrontement physique (allant de la tentative physique d'empêchement de laisser l'élève quitter la classe au point culminant qui est la violence physique de l'élève retournée sur lui-même), et d'autre part l'impact d'un usage discursif visant la réparation de la tension verbale accompagnée de geste déictique et d'un regard visant la reconnaissance de l'autre comme son interlocuteur sans visée d'affrontement. Ces résultats informent de pistes didactiques qui pourraient être mises au service de la formation des enseignants afin de leur permettre de questionner les marques conjointes et disjointes associant leur comportement posturo-mimogestuel à leur comportement verbal en situation de tension. Bien entendu, notre étude mériterait d'être étendue et confrontée à un corpus plus important, néanmoins ses résultats permettent déjà de montrer l'importance de questionner les réalisations multimodales visant une meilleure résolution des conflits en classe.

\section{Références}

Argyle, M. \& Ingham, R. (1972). Gaze, mutual gaze and proximity. Semiotica, 6(1), 32-49.

Austin, J. L. (1962). How to do Things with Words, Oxford, Oxford University Press.

Azaoui, B. (2014). Coconstruction de normes scolaires et contextes d'enseignement. Une étude multimodale de l'agir professoral. (Thèse de doctorat non publiée). Université Paul Valery, Montpellier III.

Azaoui, B. (2015a). Déritualisation et normes interactionnelles. Entre stratégie pédagogique et revendication identitaire. Bulletin Vals-Asla, Bulletin suisse de linguistique appliquée, Numéro spécial, $t$. 2. Neuchâtel: Centre de linguistique appliquée, p. 269-285. http://doc.rero.ch/record/11876/files/bulletin_vals_asla_2015_special_2.pdf

Azaoui, B. (2015b). Fonctions pédagogiques et implications énonciatives de ressources professorales multimodales. Le cas de la bimanualité et de l'ubiquité coénonciative. Revue RDLC, vol. 12 , no. 2. 2015, p. 225-254.

Brown, P. \& Levinson, S. (1987) Politeness. Some universals in language use, Cambridge, Cambridge University Press.

Calbris,G. \& Porcher, L. (1989) Gestes et communication, Paris, Didier.

Corraze, J. (1980) Les communications non verbales. Paris : PUF.

Cosnier, J. (2015). Psychologie des émotions et des sentiments. Paris : Retz (éd. originale 1994).

De Landsheere, G. \& Delchambre, A. (1979). Les comportements non verbaux de l'enseignant. Comment les maitres enseignent II. Paris : Nathan.

Flahault, F. (1978). La parole intermédiaire. Paris : Seuil. 
Foerster, C. (1990). Et le non-verbal ? in L. Dabène, F. Cicurel, M.-C. Lauga-Hamid \& C. Foerster (dir.), Variations et rituels en classe de langue (p.72-93). Paris : Hatier.

Fracchiolla, B., Moïse, C., Romain, C. \& Auger, N. (2013) «Introduction », in B. Fracchiolla, C. Moïse, C. Romain et N. Auger (dir.), Violences verbales. Analyses, enjeux et perspectives, Rennes, Presses Universitaires de Rennes.

Goffman, E. (1974) Les rites d'interaction, Paris, Éditions de Minuit.

Goodwin, C. (1981). Conversational organization. Interaction between speakers and hearers. London : Academic press.

Hall, E. T. (1971). La dimension cachée. Paris: Seuil. (éd.originale 1966)

Heylen, D. (2005). A closer look at gaze. Dans C. Pelachaud, E. André, S. Kopp \& Z.

Ruttkay (dir.), Creating bonds with embodied conversational agents : AAMAS workshop (p.3-9). Utrecht : University of Utrecht.

Kendon, A. (1967). Some functions of gaze-direction in social interaction. Acta Psychologica, 26, 22-63.

Kendon, A. (2004). Gesture : Visible action as utterance. Cambridge : Cambridge University Press.

Kerbrat-Orecchioni, C. (1992) Les interactions verbales, t. 2. Paris, A. Colin.

Krafft, U. \& Dausendschön-Gay, U. (2001). La multidimensionnalité de l'interaction. Textes, gestes et le sens des actions sociales. Marges linguistiques, 2, 120-139.

Lakoff, R. (1973) « The Logic of Politeness, or minding your p's and q's », Chicago Linguistics Society, n 9, p. 292-305.

Leblanc, S., \& Ria, L. (2014). Designing the Néopass@ction Platform Based on Modeling of Beginning Teachers' Activity. Design and Technology Education: An International Journal, ${ }^{\circ} 19$ (2). http://ojs.lboro.ac.uk/ojs/index.php/DATE/issue/view/170

Leech, G. N. (1983) Principles of Pragmatics, Londres/New York, Longman.

McNeill, D. (1992) Hands and mind : what gestures reveal about thought. Chicago : University of Chicago Press.

Moulins, J.-F. (2004). « Le discours silencieux du corps enseignant. La communication non verbale du maître dans les pratiques de classe », Carrefours de l'éducation, n 17, p. 142-159.

Pujade-Renaud, C. (1983). Le corps de l'enseignant dans la classe. Paris : ESF Editions.

Raffestin, C. (1986). Ecogenèse territoriale et territorialité. In: F. Auriac, R. Brunet (éds.) Espaces, jeux et enjeux. Paris : Fayard \& Fondation Diderot, 1986. p. 175-185

Romain, C. \& Lorenzi, N. (2013) «Interactions conflictuelles et actes de langage menaçants en classe de la maternelle au collège ", in B. Fracchiolla, C. Moïse, C. Romain et N. Auger (dir.), Violences verbales. Analyses, enjeux et perspectives, Rennes, Presses Universitaires de Rennes, p. 141-164.

Romain, C et Rey, V. (2016a). Stratégies d'affirmation et gestion (co)-énonciative de la tension verbale dans l'interaction didactique. Testi e linguaggi, Rivista di studi letterari, linguistici e filologici dell’Universita di Salerno. Vol. 10. p.143-162. 
Romain, C. \& Rey, V. (2016b). Enjeux sémantiques des actes de langage menaçants en classe : de la menace argumentative positive à la menace polémique négative. In Olga Galatanu, Ana-Maria Cozma et Abdelhadi Bellachhab (dir.), Représentations du sens linguistique : les interfaces de la complexité, (pp. 321-336). Bruxelles : Peter Lang.

Searle, J. R. (1969) Speech Acts, Cambridge, Cambridge University Press.

Watzlawick, P. ; Helmick Beavin, J. \& Jackson, D. D. (1972). Une logique de la communication, Paris : Editions du Seuil. 